\title{
Mejoramiento de las prácticas alimentarias en adultos mayores de Tumbaco
}

\section{Improvement of elderly feeding practices at Tumbaco}

\section{Yolanda Silva de Grijalva}

Carrera de Nutrición Humana, Universidad San Francisco de Quito, Cumbayá, Ecuador. ydegrijalva@gmail.com

\section{Resumen}

El proyecto de Mejoramiento de las Prácticas Alimentarias en Adultos Mayores (MEPRAM) es un estudio piloto que utilizó la metodología de consejería individual, como un aporte innovador para mejorar la calidad de vida de los adultos mayores en alimentación y actividad física. El MEPRAM fue ejecutado por estudiantes de Nutrición de la Universidad San Francisco de Quito (USFQ), como parte de sus actividades académicas y de vinculación a la comunidad. Intervino personal de la Administración Zonal de Tumbaco y adultos mayores del Programa "60 y Piquito". Los objetivos fueron mejorar las prácticas de alimentación y actividad física de 13 adultos mayores. La consejería se realizó en base a las prácticas actuales de alimentación y actividad física que realizan los adultos mayores, comparados con las prácticas ideales que recomienda la ciencia de la nutrición. Luego de la negociación individual, se aplicaron recomendaciones factibles de cambio, con un seguimiento de ocho semanas. Adicionalmente, se desarrollaron tres talleres con demostraciones para motivar a los participantes y reforzar contenidos. Los resultados revelaron el cumplimiento sostenido de las siguientes recomendaciones: comer ensaladas, disminuir la grasa, sal y azúcar, aumentar un tiempo de comida al día, mejorar la calidad de alimentos y mejorar la intensidad y duración de la actividad física. Se identificaron los puntos de resistencia y factores motivacionales que impiden o favorecen respectivamente el cumplimiento de las prácticas. El proyecto podría ser aplicado con otros beneficiarios.

Palabras clave: prácticas alimentarias, actividad física, consejería nutricional, adultos mayores.
Abstract

The project for the improvement of seniors' feeding practices (MEPRAM) is a pilot study that used the method of individual counseling as an innovative contribution to improve the quality of life of seniors associated with their diet and physical activity. As part of the academic activities and community outreach, the MEPRAM was carried out by the students of the Department of Nutrition at Universidad San Francisco de Quito (USFQ). This project also counted with the participation of the Municipality of Tumbaco and seniors of the "60 y piquito" program. The project's main objective was to improve the feeding practices and physical activity of 13 seniors. Which seniors received counseling was based on the gap between the current feeding practices and physical activity of seniors and those recommended by nutrition specialists. After the individual evaluation and bargaining, feasible recommendations for change were implemented, along with a follow-up of eight weeks. In addition, three workshops were developed in order to demonstrate and reinforce the nutrition knowledge and to motivate the seniors. The results showed compliance with the following recommendations: eat salads; reduce fat, salt and sugar in meals; increase the time allotted for one meal per day; improve food quality; and increase the intensity of physical activity. Additionally, both resistance and motivations that prevent or promote the compliance of practices were identified. This project may be replicated with other beneficiaries.

Keywords: feeding practices, physical activity, nutrition counseling, seniors. 


\section{¿A qué problema social contribuye esta investigación?}

Tos estudios de investigación sobre nutrición en las diferentes etapas del ciclo de vida, indican que existen problemas de mala nutrición, las causas de estas afecciones son múltiples y complejas. El grupo de adultos mayores (mayores de 65 años) ha sido identificado como un grupo prioritario para desarrollar programas de intervención nutricional.

\section{Introducción}

La carrera de Nutrición Humana de la Universidad San Francisco de Quito (USFQ) contempla prácticas comunitarias y vinculación a la comunidad, como una estrategia en la formación profesional de sus estudiantes. Para ello se cuenta con varios socios estratégicos: municipios, instituciones educativas y otras instituciones públicas y privadas.

La Administración Zonal de Tumbaco (AZT), que pertenece al Municipio del Distrito Metropolitano de Quito (DMQ) desarrolla proyectos comunitarios, desde hace varios años, entre los que se encuentra el Programa: "60 y Piquito" y tienen un promedio de 50 participantes que asisten a la Casa Somos de Tumbaco. El objetivo es mejorar la calidad de vida de adultos mayores.

Dentro de este programa se inserta el presente proyecto que fue desarrollado por las estudiantes de tercer semestre, en la cátedra Nutrición a través del ciclo de vida 2, de la carrera de Nutrición Humana de la USFQ, durante el período, eneromayo de 2016

El Proyecto que se denominó: Mejoramiento de las Prácticas Alimentarias en Adultos Mayores (MEPRAM), contempló una línea base para conocer aspectos generales sobre salud y nutrición de los adultos mayores (Alonso, P., Sansó, F. y Díaz-Canel, A., Carrasco, M. 2009, p. 1-14). Se realizó una encuesta de consumo de alimentos por recordatorio de 24 horas. Los resultados demostraron la existencia de malas prácticas alimentarias específicamente sobre: frecuencia (número de veces que come diariamente), cantidad (tamaño de las porciones) y calidad (contenido nutricional) de las comidas, así como una inadecuada actividad física (Ministerio de Inclusión Económica y Social. Programa Aliméntate Ecuador. 2011).
Con estos resultados, se estuvo en condiciones de elaborar un programa piloto de consejería y comunicación en Nutrición, que podría ser aplicado en audiencias (grupos objetivo) de similares condiciones (Organización Panamericana de la Salud, OPS. 2011).

\subsection{Objetivos}

El objetivo general fue mejorar las prácticas alimentarias y actividad física de 13 adultos mayores de Tumbaco y sus familias mediante el diseño e implementación de consejería nutricional interpersonal individual.

Los objetivos específicos fueron determinar las prácticas alimentarias reales de los adultos mayores, comparar con las prácticas ideales y aplicar las prácticas factibles, identificando los factores motivacionales y puntos de resistencia que permiten o impiden el mejoramiento de las prácticas. Las estudiantes desarrollarían destrezas sobre consejería en adultos mayores.

\section{Materiales y Métodos}

Los beneficiarios directos del proyecto fueron los adultos mayores que asisten al Programa "60 y piquito" de la Casa Somos de Tumbaco. La participación en el MEPRAM fue voluntaria y se limitó a 13 mujeres, una por cada estudiante. Los beneficiarios indirectos fueron las familias de las participantes, el personal de la Casa Somos y la comunidad.

Para la ejecución del proyecto, los estudiantes de la USFQ, a más de los conocimientos teóricos que han obtenido en su formación profesional, recibieron capacitación, supervisión y acompañamiento permanentes por parte de la docente (Brown, J. 2014). Además se contó con el apoyo logístico del personal de la AZT. El presupuesto lo aportó la USFQ.

\subsection{Políticas}

El proyecto se aplicó siguiendo las directrices que mantiene la USFQ para las prácticas comunitarias de sus estudiantes y para proyectos de vinculación a la comunidad, las políticas de la AZT, así como las del Programa "60 y piquito" (Ministerio de Inclusión Económica y Social, 2011). 


\subsection{Estrategias}

- El proyecto se llevó a cabo durante el periodo de enero a mayo del 2016 que corresponde al segundo semestre académico 2015-2016.

- El proyecto se realizó con los siguientes recursos: adultos mayores estudiantes y tutores de la USFQ (Carrera de Nutrición Humana).

- $\quad$ Los materiales e insumos fueron proporcionados por la USFQ.

- Todas las fases del presente proyecto fueron documentadas, con instructivos, formularios, la presentación de informes y difusión de resultados.

El Proyecto MEPRAM es un estudio de caso que aplicó una innovadora metodología de consejería nutricional personalizada que incluyó las siguientes fases:

1. Identificación de las prácticas alimentarias ideales que se realizó durante las clases teóricas de la cátedra: Nutrición a través del ciclo de vida

2. Estas prácticas fueron: consumir preparaciones blandas, en forma de puré , incluir vegetales, frutas, cereales integrales, evitar el exceso de grasas, sal y azúcar, comer al menos tres comidas al día con horarios regulares, combinar alimentos fuentes de: carbohidratos, proteínas, grasas, vitaminas y minerales, realizar actividad física regular (Restrepo, S., Morales, R., Ramírez, M., López, M., \& Varela, L. 2006)

3. Determinación de las prácticas alimentarias reales. Para ello se convocó a 13 adultos mayores que voluntariamente dieron su consentimiento informado para participar en el proyecto. Se realizó una encuesta de consumo de alimentos por recordatorio de 24 horas.

4. Comparación entre las prácticas reales e ideales. Cada estudiante con cada adulto mayor, analizaron las prácticas.

5. Identificación de las prácticas factibles y negociación para su cumplimiento.

6. Añadir prácticas sobre actividad física: 150 minutos semanales actividad física moderada aeróbicos, o bien algún tipo de actividad física vigorosa aeróbica durante 75 minutos, o una combinación equivalente de actividades moderadas y vigorosas.

7. Verificación del cumplimiento en el hogar. Se entregó a cada beneficiaria un formulario para el registro diario del cumplimiento de la (las) prácticas recomendadas (Vidal, S. V. 2013).

8. Seguimiento: se realizó cada semana durante las horas de clase de las estudiantes de enero a marzo del 2016.

9. Desarrollo de talleres con demostraciones grupales de aspectos básicos de mejoramiento de las prácticas alimentarias. Los tres talleres fueron:

- Panel sensorial: demostración de la preparación y pruebas organolépticas de la mezcla alimentaria: cereal- leguminosa denominada Fuersan.

- Mi plato: demostración para mejorar la combinación y calidad de los alimentos.

- Actividad física: demostración, calentamiento, baile terapia y relajación.

10. Evaluación: al final del proyecto piloto cada participante llenó un formulario de evaluación.

\section{Resultados}

Participaron 13 adultos mayores que pertenecen al Programa "60 y piquito", que mantiene la AZT. Las prácticas alimentarias de cada participante fueron diferentes, sea por sus condiciones de salud (hipotiroidismo, hipertensión arterial, diabetes, osteoporosis y otras), sus preferencias alimentarias, o situación económica. Las prácticas que se cambiaron en todos los casos en forma sostenida fueron: comer verduras (ensaladas), tomar desayuno, disminuir el consumo de carbohidratos, tomar líquidos, disminuir el consumo de grasas, sal y azúcar. los casos.

La aceptación y uso del Fuersan en el hogar, se cumplió también en todos 
La combinación de alimentos, para mejora la calidad de la alimentación, con el uso de "Mi plato" y la práctica de actividad física se cumplió en todas las participantes.

Los factores motivacionales que favorecieron la aplicación de las prácticas recomendadas fueron, entre otros: facilidad de la aplicación de las recomendaciones, gusto personal de los alimentos, beneficios en la salud y nutrición, aceptación familiar y de personas relacionadas con las participantes (jefes), claridad en las explicaciones por parte de las estudiantes de Nutrición.

Los puntos de resistencia, que dificultaron o impidieron el cumplimiento de las prácticas ocurrieron durante la primera semana y fueron: falta de tiempo, olvido, dietas individuales diferentes a las recomendadas (exceso de sal, azúcar y grasas, entre otros). En las semanas siguientes las estudiantes insistieron en el cumplimiento de las prácticas aplicando las técnicas de consejería.

Los resultados de las pruebas organolépticas obtenidos en el taller se presentan en las siguientes tablas. Las calificaciones fueron del 1 al 5, siendo el número 5 "excelente" y 1 "muy malo".

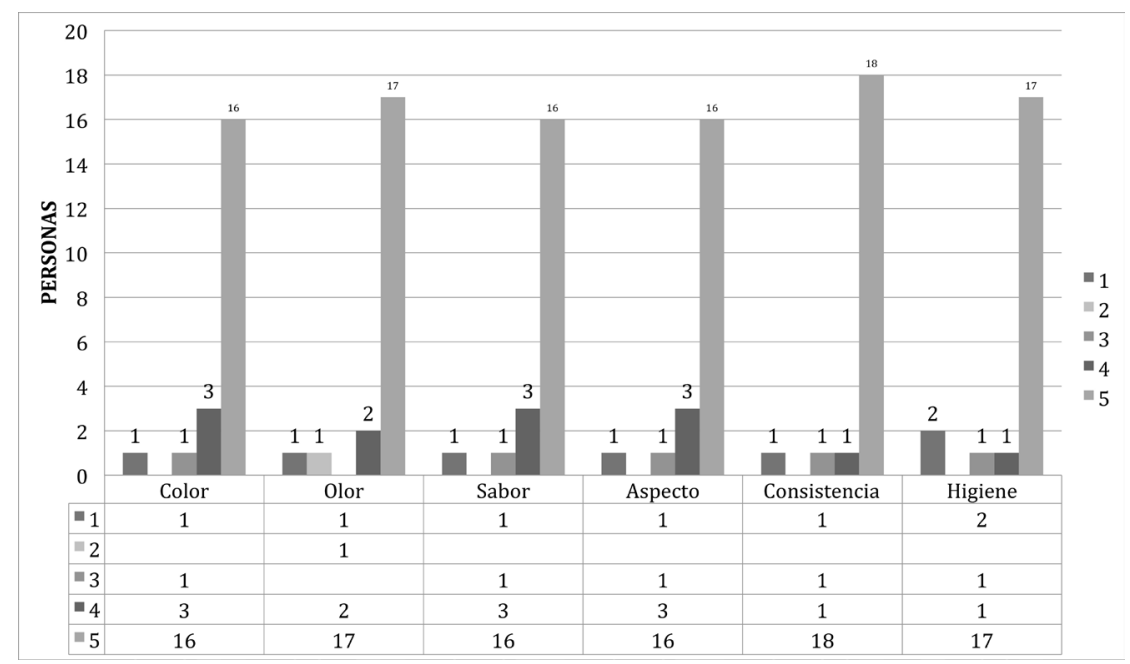

Figura 1. Niveles de aceptación del polvo Fuersan, por Encuesta sobre panel sensorial Fuersan, estudiantes de Nutrición (2016)

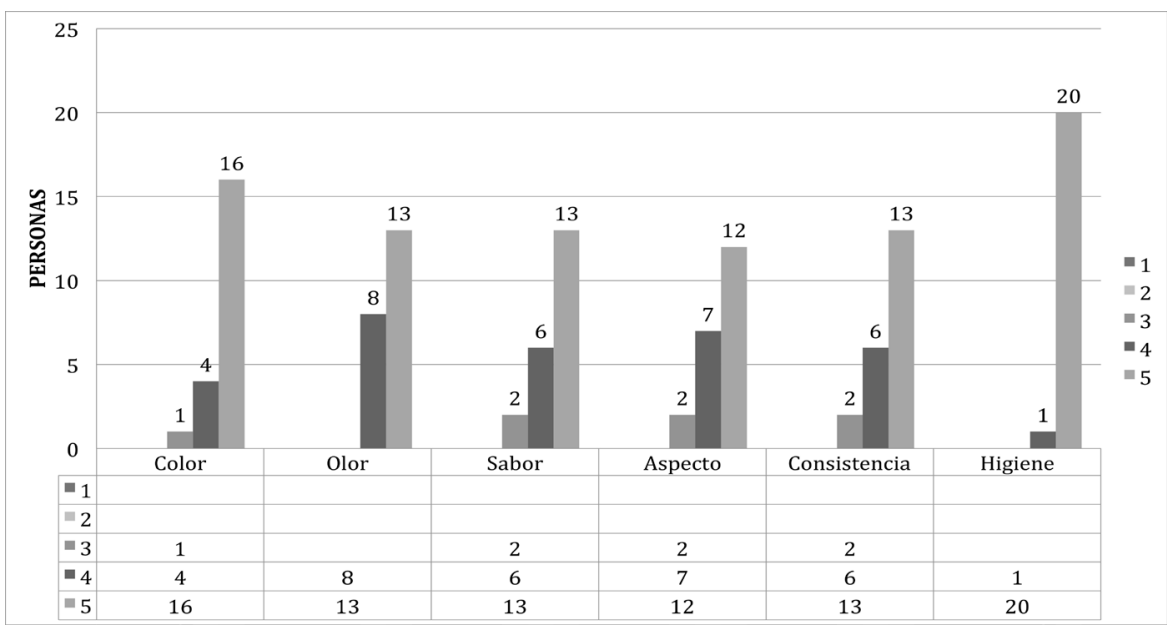

Figura 2. Niveles de aceptación del batido de piña, por Encuesta sobre panel sensorial Fuersan, estudiantes de Nutrición (2016)

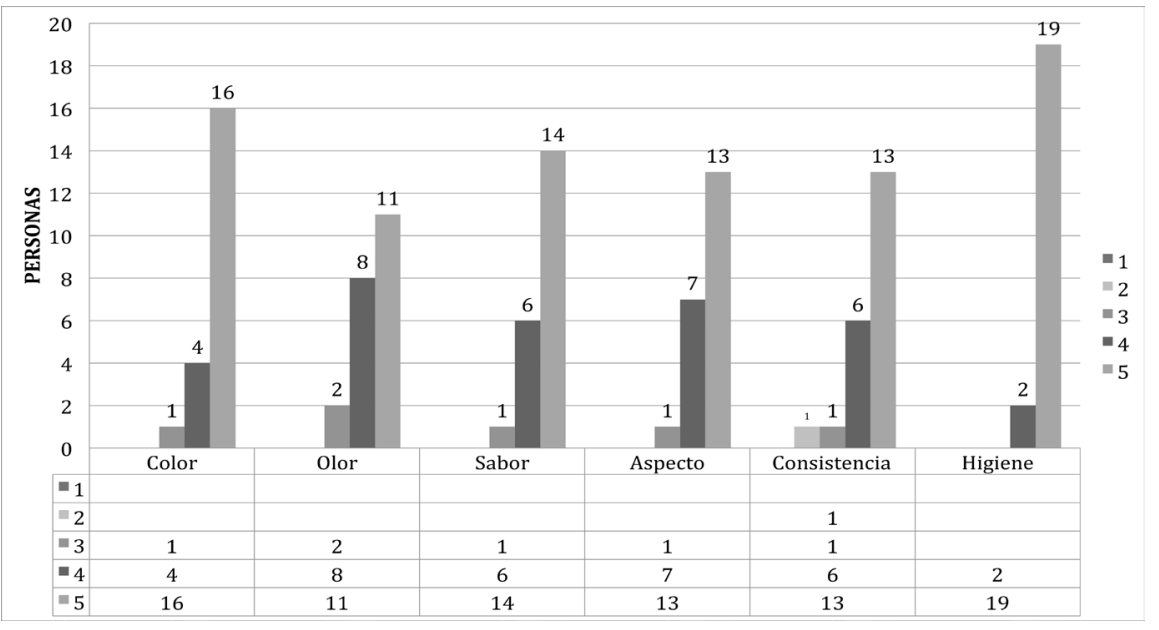

Figura 3. Niveles de aceptación del batido de naranjilla, por Encuesta sobre panel sensorial Fuersan, estudiantes de Nutrición (2016) 


\section{Conclusiones}

Las prácticas alimentarias, si bien son difíciles de cambiar en la edad adulta son factibles de mejorar con la metodología de consejería individualizada, que toma en cuenta los actuales hábitos alimentarios de los adultos mayores. Los cambios pueden sostenerse a través del tiempo.

Para el seguimiento y la sostenibilidad del mejoramiento de las prácticas alimentarias y de actividad física, es indispensable determinar los puntos de resistencia y los factores motivacionales que impiden o favorecen el cumplimiento de las recomendaciones. Estos aspectos son específicos para cada persona.

La empatía, confiabilidad, respeto y solvencia profesional del consejero nutricional es indispensable en el caso de los adultos mayores, pues son personas altamente resistentes a los cambios.

Las dificultades propias de los adultos mayores debido a su edad, enfermedades y falta de atención, pueden ser superadas con consejería individual.

\section{Síntesis y aplicaciones prácticas}

- Mejorar las prácticas de alimentación en adultos mayores determina mejores condiciones de salud.

- Las estudiantes de Nutrición Humana, están en condiciones de aplicar esta metodología en su práctica profesional privada o institucional.

\section{Referencias}

Alonso, P., Sansó, F. y Díaz-Canel, A., Carrasco, M. (2009) Diagnóstico de fragilidad en adultos mayores de una comunidad urbana. RevCub Salud Pública, 35 (2): (pp.1-14).

Brown, J. (2014) Nutrición en las diferentes etapas de la vida. Adulto mayor. McGraw Hill.

Ministerio de Inclusión Económica y Social (2011). Agenda de igualdad para adultos mayores 2012-2013. 2a ed. Ecuador.

Ministerio de Inclusión Económica y Social. Programa Aliméntate Ecuador. (2011) Encuesta Nacional de Salud, Bienestar y Envejecimiento 2010- 2011 “SABE II”. Quito, Ecuador. Incluir páginas

Organización Panamericana de la Salud, OPS. (2011). La salud de los adultos mayores. Una visión compartida. Segunda edición.

Restrepo, S., Morales, R., Ramírez, M., López, M., \& Varela, L. (2006). Los hábitos alimentarios en el adulto mayor y su relación con los procesos protectores y deteriorantes en salud (Publicación científica). RevChilNut, 33(3).

Vidal, S. V. (2013). Prevalencia de factores biopsicosociales relacionados con el proceso de fragilización en el adulto mayor cesante, en el hospital de atención integral al adulto mayor. Quito. 